\title{
EANM Focus 1: one small step for two men, one giant leap for a specialty
}

\author{
Stefano Fanti ${ }^{1}$ (D) Wim J. G. Oyen ${ }^{2,3}$ \\ Received: 22 March 2019 / Accepted: 31 March 2019 /Published online: 24 April 2019 \\ (C) Springer-Verlag GmbH Germany, part of Springer Nature 2019
}

In January 2018, the first EANM Focus meeting was held in Valencia, Spain, where we had the pleasure and honor to cochair this event. This new initiative adds to EANM's Annual Congress, that with time has evolved to the most important event in the world in the field of Nuclear Medicine, Molecular Imaging and Theranostics. Given the steadily increasing success of the EANM congress, the EANM Board fostered the idea to organize a winter event, not aiming to duplicate the Congress, but rather to cover a single topic of the highest relevance to our field. The first topic identified was prostate cancer imaging.

The design of the event proved quite straightforward; we decided that it should not become one of the many meetings where experts come and provide sometimes already wellknown presentations to an audience, rather we shifted our ambition to the organization of a consensus event, to deliver a push to the true impact on Nuclear Medicine in the field of prostate cancer imaging. The format of (e.g. NIH consensus meetings, national events on appropriate use of PET, APCCC in St. Gallen) were pivotal to design and plan the EANM Focus 1 , which expanded to multimodality imaging as well therapy.

\section{Approach - practical implications}

It was decided that the Focus 1 required a very scientific approach to consensus building. In the medical community, it is not always straightforward to perform consensus building

Stefano Fanti

fanti@aosp.bo.it

1 Medicina Nucleare, Policlinico S. Orsola, University of Bologna, Bologna, Italy

2 Department of Biomedical Sciences, Humanitas University, Milan, Italy

3 Department of Radiology \& Nuclear Medicine, Rijnstate, Arnhem, The Netherlands according to proper methodology, similar to other scientific approaches. Thus we decided to involve expert methodologists, to ensure that the product of the event would have high standards, beyond yet another workshop eventually producing some expert opinions.

This approach was also based on the observation that we should move beyond methodological guidelines. Over the years EANM had promoted a number of such guidelines, almost always mostly covering procedural issues. In a monodisciplinary environment, this is perfectly understandable and correct, and given that EANM drives the highest standard of our specialty, we have always been committed to guarantee the highest level of methods for performing and reading nuclear medicine and molecular imaging exams. In some cases, suggestions of appropriate use were incorporated, being well aware that the final decision on appropriate indication was not really in our hands. The choice to involve the most renowned experts of other specialties in Focus 1 to prepare a shared document was truly a paradigm change, especially in view of the relevance of the topic.

The need of experts from other specialties (i.e. urology, oncology, radiology, radiation oncology, pathology and others) immediately implied the building of a relevant network, which for the success of the initiative is probably as important as the robust methodology.

\section{Results - achieved milestones}

A first achievement surely was that the event was fully booked within a short period of time. Obviously, the scope of the meeting was more ambitious, with production of a manuscript to report the results of the consensus process, based on Delphi methods; the manuscript was published in Lancet Oncology December 2018 [1]. This was definitely considered an outstanding success, as it is not that frequent that papers from the nuclear medicine community are accepted by highly rated journals outside the field of nuclear medicine. 
Furthermore, the outcomes of Focus 1 confirmed that EANM, as a scientific society, should diversify its activities towards scientific support of the specialty, such as consensus on PET reporting, and others. These activities are becoming more and more relevant to enhance a reputation of reliability within the medical community.

Finally, as an almost natural consequence of the optimal collaborations with clinicians of other specialties within Focus 1 and the subsequent reinforcement of our network, there has been an increase in formal collaborations with other societies, as EANM has been invited to contribute to various initiatives, including active participation in prostate cancer guidelines, developed by the EAU (https://uroweb.org/guideline/prostate-cancer/).

\section{Future - further objectives}

In practice, part of the future has already happened. The Focus 2 event was held in January 2019, covering brain imaging in dementia with another manuscript expected to soon be ready for publication. Planning of the Focus 3 meeting is already ongoing, and will be dedicated again to oncology, in particular, diagnosis and treatment of neuroendocrine tumors. In this case, a methodology very similar to Focus 1 will be used, to arrive at a consensus report in collaboration with ENETS.

The final scope of all those EANM activities is quite simple: to obtain the full recognition of nuclear medicine as a specialty of equal importance as other scientific societies in the multidisciplinary medical community, despite being (much) smaller in size than many of our sister societies. Promotion of events with significant participation of opinion leaders of different specialties as well as the joint production of scientific papers are fundamental steps.

In this exciting era in which many new radiopharmaceuticals are being developed, it is not only important to plan studies to evaluate the effectiveness of our methods and technology, but also to plan activities that work towards their full acceptance in clinical practice.

Funding The Focus 1 event was funded by EANM.

\section{Compliance with ethical standards}

Conflict of Interest The authors declare that they have no conflict of interest.

Ethical approval This article does not contain any studies with human participants performed by any of the authors.

\section{References}

1. Fanti S, Minozzi S, Antoch G, et al. Consensus on molecular imaging and theranostics in prostate cancer. Lancet Oncol. 2018;19(12): e696-708.

Publisher's note Springer Nature remains neutral with regard to jurisdictional claims in published maps and institutional affiliations. 